\title{
DFT-based approach enables deliberate tuning of alloy nanos- tructures plasmonic properties
}

\author{
M. Bubaš, J. Sancho-Parramon \\ Ruđer Bošković Institute, Bijenička cesta 54, 10000 Zagreb, Croatia \\ Email Address: jsancho@irb.hr
}

Keywords: metal alloys, nanostructures, plasmonics, density functional theory, band structure, dielectric function

\begin{abstract}
Alloying of noble metals is lately being explored as a way to tune the optical and plasmonic properties of metal nanostructures. In order to rationally modulate properties by alloying there is a need to fundamentally understand how the composition affects them. This work demonstrates that deep insights, useful for tailoring of noble alloy plasmonic and optical properties, can be gained by a low-cost approach based on density functional theory (DFT). We show in this work that the PBE functional, commonly used for calculation of alloy optical properties, can largely underestimate and in some cases even fail to predict a plasmonic response of an alloy. We propose the use of the GLLB-SC functional as a same-cost alternative and demonstrate it has an overall better performance than PBE for alloyed nanoparticles, thin films and bulk alloys. The evolution of optical properties with composition range in the UV/Vis region is explained by connecting the alloy composition, band structure and the resulting dielectric function. Additionally, an emergent property of alloying in the form of strong optical losses due to interband transitions in the IR region is identified and its origin is elucidated.
\end{abstract}

\section{Introduction}

Nanophotonics and plasmonics have been a matter of rapidly increasing research interest over the past few decades. Due to the attractive properties that plasmonic nanostructures exhibit, such as local field enhancement, strong light absorption and scattering and efficient hot carrier generation, they are being successfully utilized for solar energy harvesting, photocatalysis, waveguiding and sensing. [1, 2, 3, 4,5] The most widely used plasmonic materials are noble and coinage metals such as $\mathrm{Au}, \mathrm{Ag}$ and $\mathrm{Cu}$ because of their strong and sharp surface plasmon resonance $[6,7,8,9]$. It is possible to tune the optical properties of nanostructures by changing their size and geometry but only within the limits imposed by intrinsic properties of the material such as electron density and electronic structure $[4,8]$.

Alloying of noble and coinage metals provides a new approach to bypass these material constraints while retaining good plasmonic properties. However, experimental determinationn of the alloy composition which results in the desired optical response can be expensive and time demanding, particularly taking into account that optical properties depend nonlinearly on the composition. Empirical models that could reduce the need of extensive experimental data are only well suited for systems such as $\mathrm{Au}-\mathrm{Ag}$, with slowly and smoothly varying optical properties and provide limited physical explanation. $[10,11]$ The importance of a deep physical insight into composition-properties relationship is in it enabling a rational tailoring of alloy properties for specific purposes.

Recently, electronic, optical and plasmonic properties of alloys have been explored using density functional theory (DFT) $[12,13]$. DFT-based approach is able to circumvent the above mentioned limitations: It requires practically no empirical data and provides a fundamental physical insight into electronic structure modification, connecting composition variation with the change in optical properties. A current issue with DFT-based approaches is finding a satisfactory combination of prediction accuracy and computational efficiency. Predicting the optical properties of different alloy compositions using DFT requires a multitude of calculations to sufficiently sample the compositional and configurational space. Calculations involving fixed empirical parameters, such as a proportion of Hartree Fock exchange, might have questionable transferability, while those involving adjustable parameters, such as DFT $+\mathrm{U},[14]$ are of little predictive use. In addition, a sufficiently large unit cell must be used for atomic ratio in a unit cell to match the desired composition, making approaches with steep scaling unfeasible. Furthermore, using hybrid functionals or applying a GW correction is of questionable effectiveness $[15,16]$ despite being order(s) of magnitude computationally more expensive than using a simple generalized gradient approximation (GGA) functional.

PBE, a GGA-level functional developed by Perdew, Burke and Ernzerhof [17] has become a standard functional for DFTbased optical properties calculation [18, 19, 20, 21, 22, 23, 24, 25, 26, 27]. It is ubiquitous in solid state DFT [28, 29, 30, 31] due to its universality, computational efficiency and well understood physical basis with no empirical parametrization. However, PBE has known limitations pertaining to d-band treatment in different materials. This results in considerable underestimation of band gaps and interband transition energies [32, 33, 34].

The aforementioned problems show that the desired DFT approach should ideally involve a functional with similar advantages as PBE but better in predicting optical properties. GLLB-SC functional potentially meets these requirements, as has been recently shown in calculations for gold and silver [33, 34, 35, 36]. GLLB-SC functional uses a GLLB exchange response potential developed by Gritsenko et al. [37] and PBEsol functional for energy density and correlation. GLLB potential allows a very efficient evaluation of exchange discontinuity contribution to quasiparticle band gap. Discontinuity contribution widens the gap and counteracts the interband transitions energy underestimation typical for GGA functionals, while remaining comparably efficient and not reliant on parametrization. Moreover, the formulation of GLLB potential is 
based on physically grounded arguments such as proper asymptotic behavior, exchange scaling relation and fit to the homogenous electron gas [38].

In this work we present a universal method suited for high throughput calculations of the optical response of plasmonic alloys. Accurate results were obtained for structure optimization, band structure, and consequently, optical and plasmonic properties of several binary alloys of noble and coinage metals. We compare bulk dielectric functions for pure elements calculated by several functionals and show that GLLB-SC has the overall best performance. For alloy systems, we compare in depth the performance of PBE and GLLB-SC in the UV and visible range exploring their different d-band treatment. Next, we focus on the energy range below the nominal d-sp transition onset and show the emergence of optical losses caused by interband contribution to dielectric function upon alloying. Calculated dielectric functions allow us to predict the plasmonic response of nanoparticles and films and compare them to predictions based on experimental data. Finally, we discuss the implication of our results for practical applications and the validity of the presented calculations scheme as a generalpurpose approach for ab-initio calculation of optical response of plasmonic alloys.

\section{Results}

We considered four binary alloy systems, $\mathrm{Au}-\mathrm{Ag}, \mathrm{Au}-\mathrm{Cu}, \mathrm{Ag}-\mathrm{Cu}$ and $\mathrm{Au}-\mathrm{Pd}$. Constituent elements form bulk crystals with FCC lattice, and substitutional alloys with FCC-like lattice in all of the aforementioned binary systems. Compositional space was sampled with increments of $12.5 \%$, which made for a total of 9 different compositions for each alloy system. 4 atom cells were used for pure elements and alloys of $25 \%, 50 \%$ and $75 \%$ composition ratio. 8 Atom cells were used for $12.5 \%, 37.5 \%, 62.5 \%$, and $87.5 \%$ composition ratio. We found 3 different possible configurations of atomic ratio $3 / 8(37.5 \%)$ and $5 / 8(62.5 \%)$, all of which were included in calculations for the purpose of studying the compositional influence on dielectric function. The configurations for $\mathrm{Au}_{5} \mathrm{Ag}_{3}$ can be seen on Figure S1 (a).

\subsection{Structure optimization}

An indirect way to ensure the quality of calculated optical properties is by proper lattice optimization, as dielectric function can be affected by lattice parameters of optimized structure. Works of Haas et al. [39] and Csonka et al. [40] show that PBEsol performs well for lattice constant determination of most bulk solids, especially for pure noble metals. We calculated the lattice constants with both PBE and PBEsol for pure metals used in this study. PBEsol performed better than $\mathrm{PBE}$ for bulk $\mathrm{Au}, \mathrm{Ag}$ and $\mathrm{Pd}$ lattice constant calculations, while for $\mathrm{Cu}$ their performance was comparable, as shown in Table S1. Therefore, all alloy structures were optimized using PBEsol. The choice of optimization method can be important as different lattice constant can noticeably influence the shape of dielectric function as can be seen on Figure S2 for the imaginary part of the dielectric function $\left(\varepsilon_{2}\right)$ of $\mathrm{Ag}$ based on PBE and PBEsol lattice constants.

\subsection{Electronic structure and optical calculations}

\subsubsection{Comparison for pure elements}

The dielectric function can be separated into contributions of interband and intraband transitions $\varepsilon(\omega)=\varepsilon_{\text {intra }}(\omega)+$ $\varepsilon_{\text {inter }}(\omega)$, where the effect of intraband transitions is introduced via phenomenological Drude term, as implemented in GPAW code:

$$
\frac{\omega_{p}^{2}}{(\omega+i \gamma)^{2}}
$$

where $\omega$ is the light frequency, $\omega_{p}$ is plasma frequency and $\gamma$ is the scattering rate parameter, not calculated ab initio but set usually to $0.01 \mathrm{eV}$ in calculations, or adjusted to better match experimental values. In the IR energy region, for closed $\mathrm{d}$-shell elements such as $\mathrm{Au}, \mathrm{Ag}$, and $\mathrm{Cu}$, contribution by interband transitions is small to non-existent and contribution by intraband transitions dominates. Position of the d-bands for these elements is well below the Fermi energy, so energy of transitions from d-bands to sp-conduction band falls into visible and UV range. For Pd, an open d-shell element, the two contributions are comparable in NIR due to d-bands crossing Fermi energy, thereby allowing low energy interband transitions to occur near the Fermi energy. Comparison of dielectric functions obtained by calculations performed using several different computationally efficient functionals (up to GGA-level) shows that all functionals perform similarly well for Pd but for $\mathrm{Au}, \mathrm{Ag}$ and $\mathrm{Cu}$ some of them perform quite differently, especially when predicting interband transition onset (Figure 1). To quantify the performance of each functional we compared the interband transition onset estimated from calculated dielectric functions with the interband transition onset estimated from experimental data. For that purpose we defined the interband transition onset as the energy after $\varepsilon_{2}$ minimum at which $\varepsilon_{2}$ reaches $200 \%$ of its minimum value. Data in Table S2 shows that GLLB-SC has by far the most correct estimations of interband transition onset, with almost perfect agreement for $\mathrm{Au}$ and $\mathrm{Cu}$ and slightly underestimated for $\mathrm{Ag}$. For all other functionals the interband onset is substantially red-shifted for each metal. 
Since all the functionals except GLLB-SC give similar results as PBE we proceed by performing all the following calculations using only PBE, as the current standard, and GLLB-SC as the best candidate for a new standard, based on calculations of dielectric function for pure plasmonic metals.
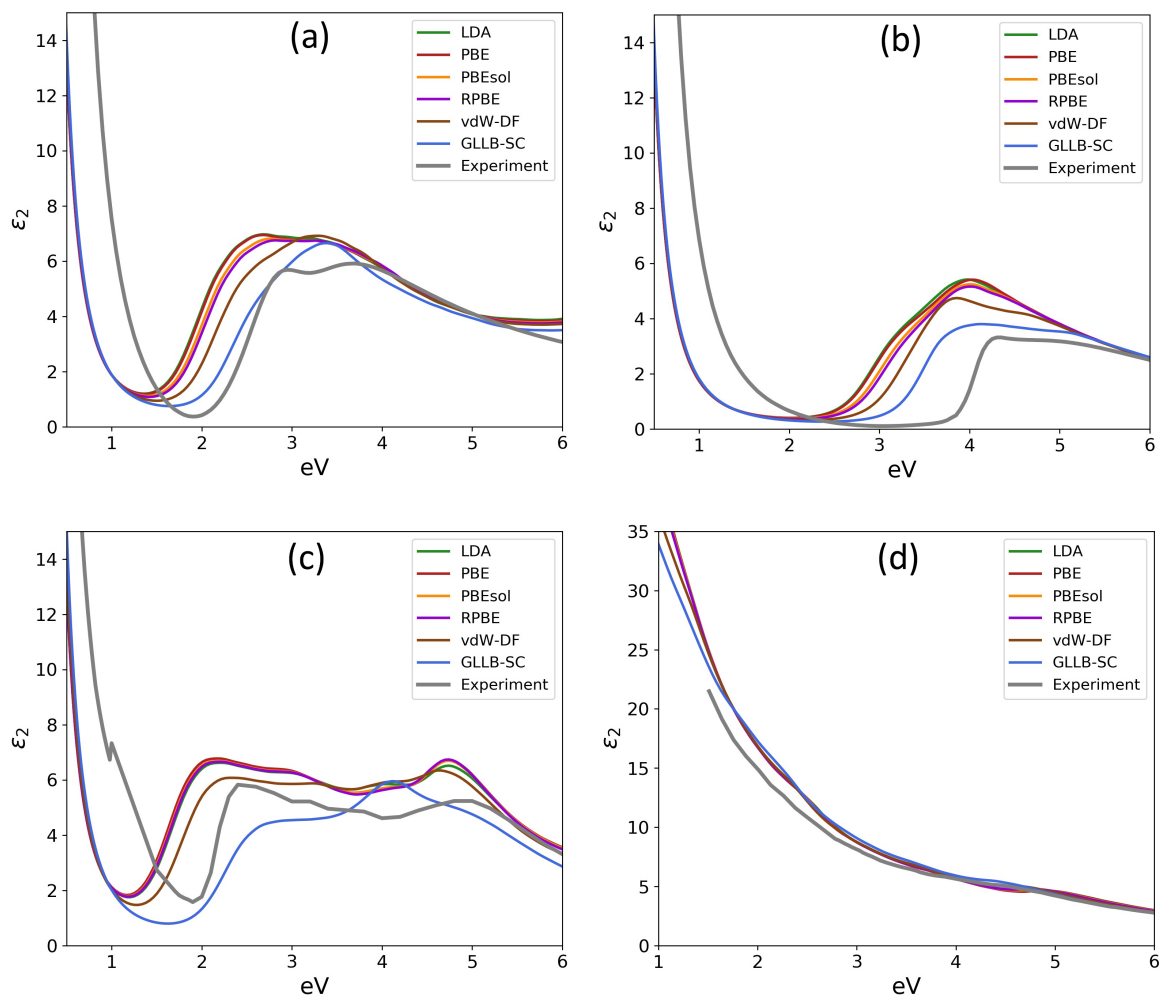

Figure 1: Comparison of imaginary part of dielectric function calculated with several GGA-level functionals (LDA [41], PBE [17], PBEsol [42], RPBE [43], vdW-DF [44], GLLB-SC [38]) with experimental data for Au (a), Ag (b), Cu (c) and $\mathrm{Pd}(\mathrm{d})$. The discrepancy in the values of $\varepsilon_{2}$ below interband transition onset between DFT and experimental data is because the scattering rate $\gamma$ was set to $0.01 \mathrm{eV}$ for all elements instead of being adjusted to better agree with experiments. To isolate the effects of different functionals on the calculated optical properties, calculations were performed using systems with experimentally determined lattice constants (Table S1). Experimental data for dielectric functions is from [45] for Au and $\mathrm{Ag}$, from [46] for $\mathrm{Cu}$ and from [47] for $\mathrm{Pd}$.

Band structure calculations (Figure S4) show that more accurate estimation of interband transition onset energy with GLLB-SC is mostly due to better treatment of d-bands, which for PBE lie too close to the Fermi energy. In GLLB-SC calculations d-bands of $\mathrm{Au}, \mathrm{Ag}$ and $\mathrm{Cu}$ are positioned lower, while other bands align more closely with $\mathrm{PBE}$ bands. For $\mathrm{Pd}$ d-bands are very similar for both functionals, as is the dielectric function. Similarly to the effect of Hubbard-like U applied to d-bands, lower position and width of GLLB-SC d-bands can be attributed to more localized d-orbitals than PBE predicts. We note in passing that comparison of GLLB-SC with several DFT $+\mathrm{U}$ calculations with varying $\mathrm{U}$ value shows $\mathrm{PBE}+\mathrm{U}$ dielectric function becomes rather similar to GLLB-SC one at $\mathrm{U}=2 \mathrm{eV}$ for $\mathrm{Au}$ and $\mathrm{U}=3 \mathrm{eV}$ for Ag (Figure S1). There is a fundamental basis to this similarity. The U parameter introduces a discontinuity in the potential acting on the orbitals which it is applied to. If these orbitals are involved in interband transitions (as is the case with d-orbitals for d-sp interband transitions), a discontinuity introduced in the exchange-correlation potential by a properly chosen $U$ value adds to the gap between d-band and sp-band at a given k-point, which results in increased interband transitions energy [48]. The effect of discontinuity introduced by $\mathrm{U}$ parameter can, therefore, be quite alike to the effect of eigenvalue-dependent discontinuity inherent to GLLB-SC [38]. Since GLLB-SC is based on PBEsol, we also performed a PBEsol+U dielectric function calculation for $\mathrm{Ag}$ using the $\mathrm{U}$ value of $3 \mathrm{eV}$ applied to d-orbitals. The obtained results are in very good agreement with both $\mathrm{PBE}+\mathrm{U}$ and GLLB-SC dielectric function (Figure S5).

\subsubsection{Comparison of dielectric functions for alloys}

\section{High energy d-sp interband transitions}

Dielectric functions of all alloy compositions are shown on the Figure 2. In the case of compositions for which multiple configurations were created, dielectric function on Figure 2 is the average of all configurations. For $\mathrm{Au}-\mathrm{Ag}, \mathrm{Au}-\mathrm{Cu}$ and $\mathrm{Ag}-\mathrm{Cu}$ systems, in which both components have closed d-shells, it can be noticed that interband transitions define almost completely the dielectric function shape in the visible/UV range. A relatively smooth trend in optical properties variation 

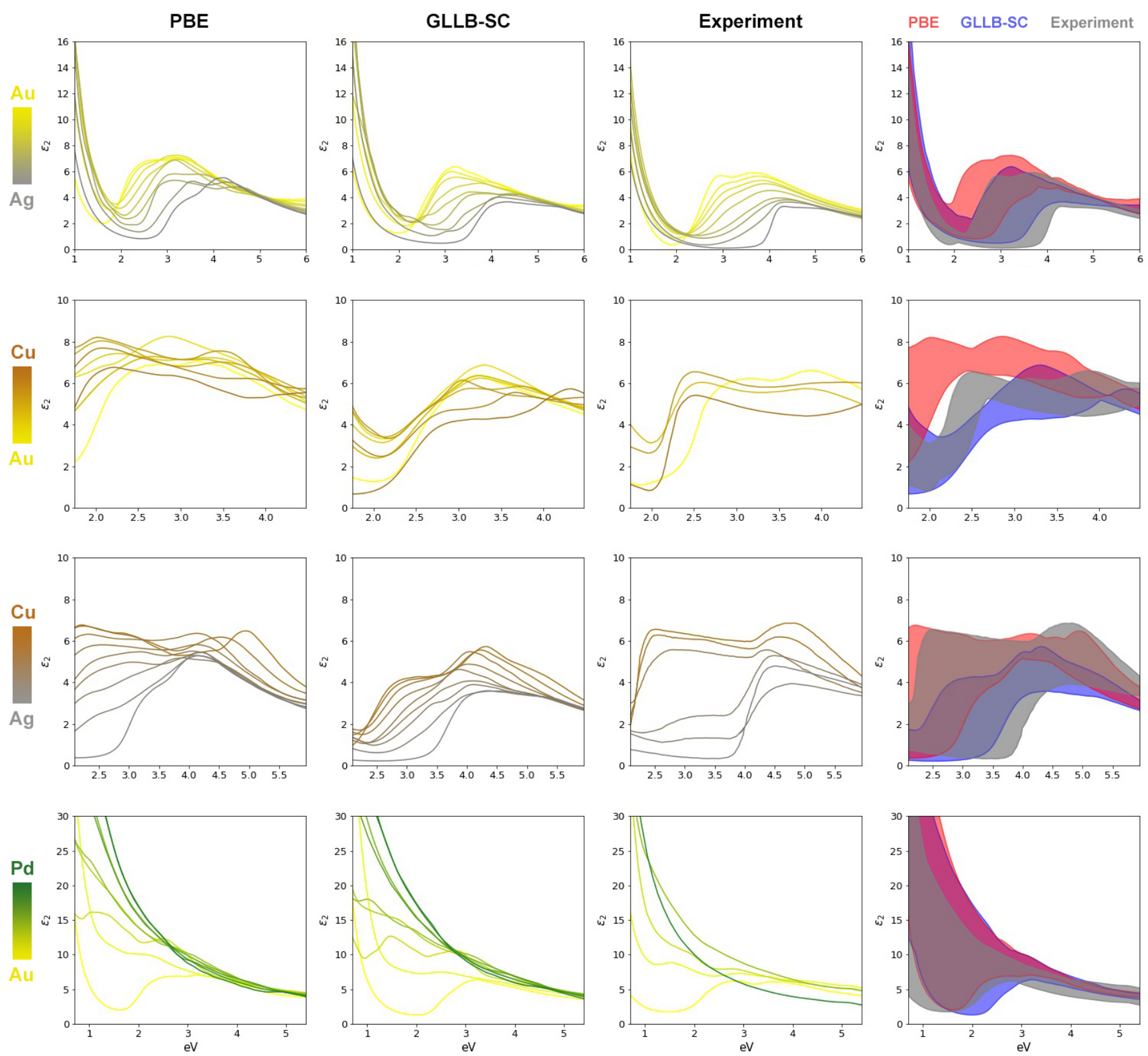

Figure 2: Imaginary part of alloy dielectric functions shaded by changing composition calculated with PBE (leftmost column) and GLLB-SC (middle-left column), experimentally determined (middle-right column) and their respective envelopes determined by minimum and maximum value of $\varepsilon_{2}$ at a given photon energy (rightmost column). Dielectric function is shaded progressively more yellov for increasing $\mathrm{Au}$ content, brown for $\mathrm{Cu}$ content, gray for $\mathrm{Ag}$ content and dark green for Pd content. The envelope is shaded blue for GLLB-SC, red for PBE and gray for experimental data. Experimental data is from [45], for $\mathrm{Au}-\mathrm{Ag}$, from [49] for $\mathrm{Au}-\mathrm{Cu}$, from [50] for $\mathrm{Ag}-\mathrm{Cu}$ and from [51] for $\mathrm{Pd}-\mathrm{Au}$ alloy. $\varepsilon_{1}$ plots can be found in Figure S6 
with change of composition has qualitatively been reproduced by both GLLB-SC and PBE. Smooth transition of d-sp onset follows the variation in d-bands position (Figures S11, S12, S13, S14). Since both functionals reproduce the smooth variation of optical properties relatively well, their comparative performance is largely determined by the limiting cases in the trend, which are pure metals. Therefore, for alloys, as was the case for pure metals, values of $\varepsilon_{2}$ in the UV/Vis region calculated with GLLB-SC are relatively close to experimentally determined values, while with PBE they tend to be systematically overestimated. For both functionals, prediction of intricate shape features varies from element to element. For example, unlike GLLB-SC functional, PBE captures double-peaked shape of Au dielectric function, while for Ag the shape of GLLB-SC $\epsilon_{2}$ agrees much better with experiments than is the case with PBE. For practical applications, however, such discrepancies in shape features are not as important as the correct position of interband transition onset, because a shift of transition onset shifts the entire function and creates far larger discrepancies in $\epsilon_{2}$ values.

Unlike the smooth variation present in systems composed of only closed d-shell elements, Au-Pd system shows an abrupt change in dielectric function on a small addition of open d-shell Pd. Both functionals predicted this highly nonlinear behavior. Band structures of Pd-Au alloy system in Figure S13 show that, compared to pure Au band structure, even the alloy compositions with the smallest proportion of $\mathrm{Pd}$, have d-bands significantly shifted to higher energies and several close lying bands crossing Fermi level and enabling interband transitions.

Configuration influence on dielectric function shape at a given composition has shown to be relatively small in the UV/Vis region. The configuration in which atoms of two elements are the most segregated exhibits a slight deviation in the UV/Vis region from the other two configurations in which atoms of two elements are more intermixed, and whose dielectric functions are practically overlapping. An example in the case of $\mathrm{Au}_{5} \mathrm{Ag}_{3}$ can be seen in Figure S3 in Supporting information.

\section{Low energy interband transitions}

Although less pronounced, increase of the imaginary part of dielectric function in the NIR region is also, rather unexpectedly, present for the systems composed of closed d-shell elements with low-lying d-bands. The increase can be almost fully ascribed to the contribution of interband transitions, as evidenced in Figure 3. Moreover, $\varepsilon_{2}$ interband contribution extends deeper into the IR region, to the edge of far infrared, and reaches much higher values than in the UV and visible region. Despite $\varepsilon_{2}$ interband values being higher at very low energies relative to UV or Vis region, they are overshadowed by even higher intraband contribution (Drude term) in low energy region $(3,(b))$. It is important to note that low energy interband contribution to dielectric function is present for all of $\mathrm{Au}, \mathrm{Cu}$ and $\mathrm{Ag}$ alloy systems and compositions, but for none of the pure constituent metals, which makes them an emergent property of alloying. We argue in the Discussion section that this emergent property is not a result of $d$-sp transitions, which are present in the UV and Vis range, but rather comes about from conduction band splitting upon alloying.

The effect of configuration on the interband transitions contribution to dielectric function in the IR is much more pronounced than in the UV/Vis region (Figure S1).

(a)

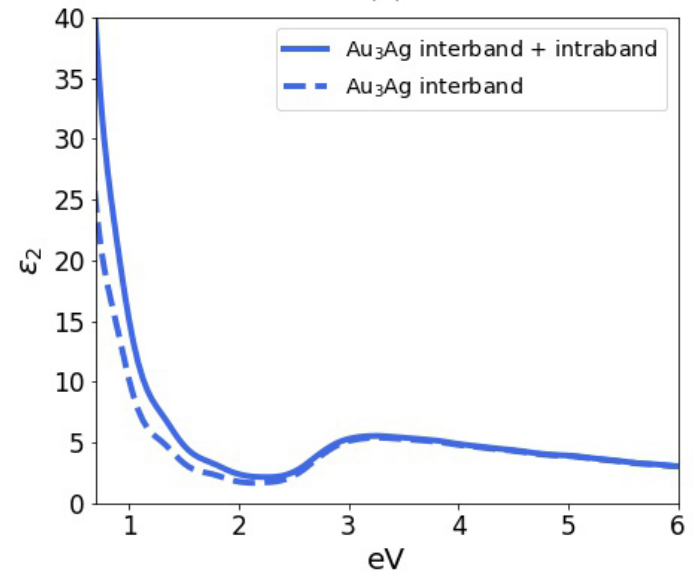

(b)

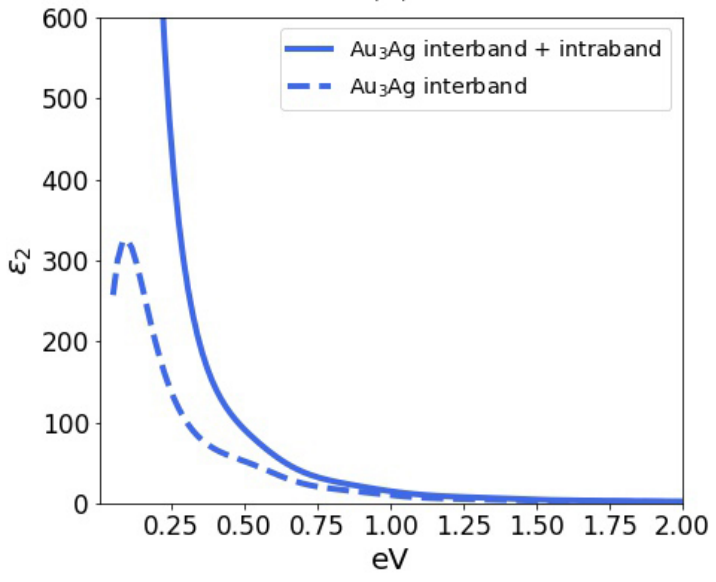

Figure 3: Imaginary part of $\mathrm{Au}_{3} \mathrm{Ag}$ dielectric function calculated using GLLB-SC with both interband and intraband contribution included (full line) and with only interband contribution (dashed line). Interband contribution is dominant even below the nominal d-sp interband transition onset (a), but is overpowered by intraband (Drude) contribution in the midand far-infrared region despite taking far larger values than in the UV/Vis region (b).

\section{Electrodynamics simulations of alloys plasmonics}

In this section we evaluate the ability of computed dielectric functions to predict the plasmonic response of alloys. Localized surface plasmon resonances are first investigated in the small particle limit by calculating the dipolar polarizability of spherical particles in the quasi-static approximation (first row in Fig. 4). Generally speaking, both experimental and 
computed dielectric functions show a progressive red shift and broadening of the localized surface plasmon resonance as the composition changes from Ag to Au. However, the experimental data predicts narrower peaks with larger polarizability values compared to those provided by computed dielectric functions, especially for Ag-rich alloys. In this regard, the GLLB-SC functional provides a better agreement with experimental data than PBE. The resonance central energy computed by GLLBS-SC is blue-shifted about $0.1 \mathrm{eV}$ with respect to the values calculated using experimental data, while PBE results are red-shifted by $\approx 0.5 \mathrm{eV}$.

Regarding field enhancement effects here we consider the case of a dimer of closely located particles excited by light polarized along the dimer axis. This configuration results in a hot-spot with large field enhancement at the gap separating the particles (Fig. 4, second row). The near-field intensity spectra computed using PBE data is red-shifted about $0.2 \mathrm{eV}$ with respect to those computed using experimental data, while GLLB-SC is $\approx 0.1 \mathrm{eV}$ blue-shifted. In a similar way as in the previous case, the field enhancement is underestimated using DFT dielectric functions, with GLLB-SC results being closer to those computed from experimental data.

As a last case-study of localized plasmon resonances we focus on nanorods as a well-studied geometry that enables tuning the resonance energy by controlling the particle aspect ratio. The extinction cross section for energies around the dipolar longitudinal resonance of a nanorod with aspect ratio 8 is shown in Figure S15. A weak spectral variation of the plasmon resonance with the composition is revealed by electrodynamics simulations based on experimental dielectric functions. This plasmon resonance variation appears to be better depicted when using GLLB-SC rather than PBE dielectric function based simulations. Compared to the previous cases, the width and strength of the localized surface plasmon resonance computed using DFT is in closer agreement with experimental data.

Next we move to the study of surface plasmon polaritons in extended systems by first looking at the dispersion relation of surface waves at a metal-glass interface. The relation between wave frequency and the real and imaginary part of the wave propagation constant is shown in Figure 4 (third and fourth rows). It can be seen that the experimental data provides a dispersion relation that is close to an ideal lossless system in which lower and upper branches, describing bound and propagating modes respectively, can be clearly distinguished (third row). These branches are separated by a region with large imaginary part of the propagation constant (fourth row) that quenches the propagation length of the mode. This description becomes less ideal for Au-rich alloys, due to the larger losses. In the case of DFT computations, the dispersion relation is smoothed out and the distinction between propagating and bound modes and the existence of a forbidden propagation region is less evident. Yet, it should be noted that results using GLLB-SC are closer to the ideal lossless metal system and the frequency regions defining bound, forbidden and propagating waves are in much better agreement with the experimental-based calculation than in the case of PBE.

We now consider the Kretschmann configuration for exciting surface plasmon polaritons for sensing purposes. A system consisting of glass (prism)/ $40 \mathrm{~nm}$ film/ air is assumed and the reflectance of light coming from the glass side as function of the incident angle is computed for monochromatic red light (fifth row in Fig. 4). A dip on the angular-dependent reflectance is observed in all cases, that becomes broader and shifted to larger angles as the amount of Au in the alloy increases. The calculations using PBE predict much wider peaks and a larger variation of the dip with the composition when compared to calculations based on GLLB-SC and experimental data, that are in much better mutual agreement. Since the width of the reflectance dip ultimately defines the figure of merit of the sensing scheme, PBE calculations can significantly underestimate the performance of the Kretschmann configuration for Au-rich alloys.

Finally, the reflectance of an air-metal interface at normal incidence as a function of photon energy is also calculated (Figure S16). Although no surface plasmons are excited in these scheme, this quantity is important to predict the color of metals and the performance of inferential systems with metal layers. The spectra is separated in a low-energy high-reflecting region, dominated by the Drude contribution and a high-energy low-reflecting region, where the optical properties are related to the interband electronic transitions. The effective plasma frequency determines the passage between these regions. A significantly better agreement of these spectral regions limits between experimental-based and DFT-based data computations is obtained using the GLLB-SC functional, in the line as in previous cases.

\section{Discussion}

Along with the ability to predict optical properties with little to no experimental data, a crucial value of a DFT-based approach lies in the fundamental physical insight that helps explain the obtained data and draw further conclusions about useful properties of the system. For example, the abrupt change in dielectric function upon alloying a small amount of $\mathrm{Pd}$ with Au was successfully predicted and also explained by changes in the band structure. DFT calculations with both functionals are able to predict this behavior with no empirical input, while it would likely take both fabrication and measurement of many samples to create empirical models that properly predict such behavior. The reason for the abrupt increase in $\varepsilon_{2}$ values in the NIR region upon alloying Au with $\mathrm{Pd}$ is the introduction of high-lying $\mathrm{Pd}$ d-states in the system. $\mathrm{Pd}$, being an open d-shell element, has d-bands crossing the Fermi level, which enables interband transitions continuously from IR to UV. This property is transferred to the Pd-Au alloy by $\mathrm{Pd}$ atoms introducing high-lying d-states in the alloy. Thus, the abrupt change in dielectric function for Pd-Au alloys arises as an inherent property of one of the alloy's constituent elements. Another example of the added value is a very important finding regarding introduction of low energy interband 

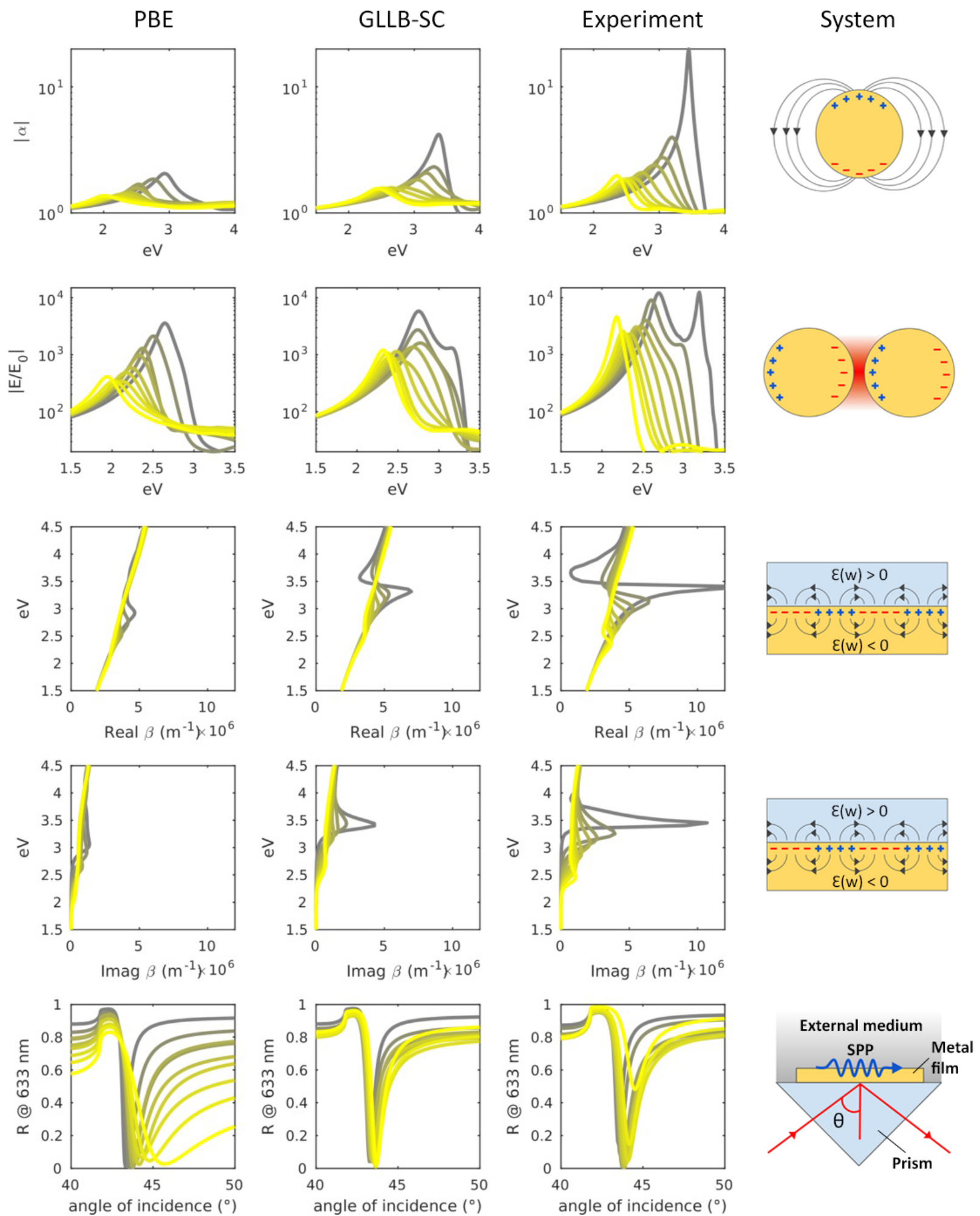

Figure 4: Plasmonic response of different systems as a a function of composition, computed using dielectric functions obtained with PBE (leftmost column) and GLLB-SC (middle-left column), and experimentally obtained dielectric functions (middle-right column). Top row: absolute value of particle polarizabilty of small spherical particles in vacuum. Second row: near-field intensity enhancement at the gap between two particles with radius $20 \mathrm{~nm}$ and separated by $5 \mathrm{~nm}$ excited by polarization parallel to the dimer axis placed in water. Third and fourth rows: real and imaginary part of the dispersion relation of a glass/metal film interface. Fifth row: reflectance of a glass/metal/air system at $633 \mathrm{~nm}$ as a function of the incidence angle. 
transitions as an emergent property upon alloying closed d-shell elements, manifesting as a considerable contribution to dielectric function in the IR region. As discussed below, band structure calculations also helped elucidate the origin of these transitions, as well as the reason why they are not present in the pure $\mathrm{Au}, \mathrm{Ag}$ and $\mathrm{Cu}$. Unlike in $\mathrm{Au}-\mathrm{Pd}$ system, in $\mathrm{Au}-\mathrm{Cu}$, $\mathrm{Au}-\mathrm{Ag}$ and $\mathrm{Ag}-\mathrm{Cu}$ alloys both elements are closed d-shell elements, so the alloy d-bands are positioned below Fermi level. Thus, the introduction of high-lying d-bands could not be the reason for the emergence of lower-energy interband transitions.

Substitution of an atom in a pure system unit cell for an atom of another element introduces a perturbation that breaks the degeneracy in the band structure. Degeneracy breaking results in "splitting" of conduction band, which is visible in the band structures of alloys. Nearly parallel bands close in energy, crossing the Fermi level, introduce new interband transitions of the appropriate (low) energy. This finding is corroborated by experimental ellipsometric data of ordered $\mathrm{AuCu}_{3}$ [52] for which a sharp $\varepsilon_{2}$ peak appears between around $1 \mathrm{eV}$. This peak in $\varepsilon_{2}$ is not present for the disordered system of the same composition, which points to stronger configurational dependence of dielectric function at low energies, also predicted by DFT. It is likely that, in disordered systems, averaging over many different local environments smooths out the sharp features in low energy dielectric function. This could explain why sharp features are not present for the $\mathrm{Au}-\mathrm{Ag}$ alloy system, which is a solid solution in the entire compositional range. Although some sharper features may be sensitive to configurational change, the overall interband contribution to dielectric function in the lower energy region is quite consistent for a given composition. Interband contribution to $\varepsilon_{2}$ is especially pronounced in NIR where it amplifies optical losses, especially for compositions close to 50/50 ratio. Increased optical losses in the IR region for $\mathrm{Au}$ - $\mathrm{Ag}$ alloys have been thoroughly discussed in the literature, with the likely cause ascribed to increased scattering due to lattice distortions, impurities and other imperfections.[11, 45, 53, 54] We propose low-energy interband transitions as an emergent alloy property that will contribute to the IR absorption of materials regardless the structural non-idealities. A potentially very important utilization of emergent low energy transitions in plasmonic alloys is for efficient hot electron generation. According to the work of Brown et al. plasmon decay due to interband transitions is the most efficient for hot electron generation among several plasmon decay mechanisms.[14] Their calculations show that a significant portion of hot electrons generated by plasmon decay for pure gold at $2 \mathrm{eV}$ is due to interband transitions, even though minimal interband transitions are available at that energy. At higher energies interband transitions strictly dominate the hot electron generation. As alloying causes several times higher interband contribution than for pure plasmonic metals below $2 \mathrm{eV}$, alloyed plasmonic nanoparticles could have much more efficient hot electron generation in the visible and NIR range. By changing the size, shape and composition, $\varepsilon_{2}$ could be tuned to a "sweet spot" value at plasmon frequency, where $\varepsilon_{2}$ is low enough not to overly dampen the plasmon peak, but has a high enough interband transitions contribution to generate hot electrons very efficiently. The plasmonic response of different systems calculated using DFT data qualitatively agrees with that based on experimental data, in all cases correctly predicting the broad trends of angular and photon energy dependence of the plasmon resonance. As expected from theoretical consideration regarding discontinuity contribution implemented in the GLLB-SC functional, it performs better than PBE, especially when Au-rich alloys are considered. The quantitative agreement depends on the specific system being considered. For those configurations showing resonances in the visible part of the spectra the optical properties are dominated by d-sp transitions that, as shown before, are best accounted by the GLLB-SC functional. In some situations the losses overestimation given by PBE results in a nearly complete quenching of the plasmonic response, like in the case of small particles and the dispersion relation of Au-rich alloys. If the resonance, however, takes place in the infrared part of the spectra like in the case of nanorods, the free electron Drude contribution dominates and the response is less sensitive to losses coming from interband transitions. However, it should be noted that DFT calculations correctly predict a damping of the plasmon resonance for alloys in comparison to pure materials regardless whether the damping term of the Drude model is set equal to $0.01 \mathrm{eV}$ for all compositions. The reason is the previously described low-energy interband transitions that can partially account for the experimentally observed enhanced infrared losses in alloys.

The PBE shortcomings described above are a critical obstacle for satisfactory description of optical properties on a nanoscale in the operational spectral range for most plasmonic applications. Particularly, it turns out that PBE is unsuitable for prediction of optical properties of Au-Ag alloy nanoparticles and casts doubt on its reliability for prediction of nanophotonic properties for similar systems. Conversely, interband contribution to bulk dielectric function in UV and visible range, as predicted with GLLB-SC, is in much better agreement with experimental data. Due to the relatively small influence of configuration on dielectric function, taking into account only one unit cell configuration might be sufficient. For disordered alloys, most likely a configuration with diverse local atomic environments would be a preferable choice.

\section{Conclusion}

We have shown that optical and plasmonic properties of alloy nanostructures can be qualitatively predicted by a DFTbased approach. Results obtained by calculations with GLLB-SC functional are overall more accurate than results obtained by calculations with standardly used PBE functional. Since GLLB-SC has a well grounded physical basis and same cost as PBE, which makes it suitable for high throughput calculations, we propose it as a new standard for predicting plasmonic properties of alloys. 
As a significant benefit of such DFT-based approach, the insights gained by DFT in our work provided a causal link between different changes in band structure and their effect on the dielectric function. In the UV and visible range a smooth variation of optical properties with changing composition can be explained by shifting of the d-bands which changes the d-sp transition onset. In the IR range we identified the occurence of optical losses caused by interband transitions as an emergent property of alloying which could have importance in hot electron generation. We showed that these losses are caused by closely-lying conduction bands present in all studied alloy nanostructures. The effect of configuration change was shown to be minor in the UV and visible range and more pronounced in the IR range.

These findings pave a way to deliberate band structure engineering for desired purposes from IR to UV range and introduce a possibility of a novel approach to efficient hot electron generation.

\section{Methods}

DFT calculations were performed using GPAW package [55, 56], based on the projector augmented wave (PAW) method and the atomic simulation environment (ASE) [57]. Brillouin zone sampling was performed using Monkhorst-Pack grid [58], plane waves were used as a basis set, and occupation number smearing was of Fermi-Dirac type with $0.1 \mathrm{eV}$ width.

\subsection{Structure optimization}

Structures of pure metals were optimized both by PBE [17] and PBEsol, [42] which has been shown to perform well for calculations of lattice parameters for a large range of materials [39, 40]. PBEsol performed better for bulk $\mathrm{Au}, \mathrm{Ag}$ and $\mathrm{Pd}$ lattice constant calculations, while for $\mathrm{Cu}$ their performance was comparable. (Table in supplementary material). Hence, all alloy structures were optimized using PBEsol functional. 24x24x24 k-points were used for 4-atom cells and 24x24x12 $\mathrm{k}$-points for 8-atom cells. Since length along the z-axis in real space is approximately twice as long than along the $\mathrm{x}$ - and $\mathrm{y}$-axis for 8-atom cells, in the reciprocal space along the z-axis they are one half of the length along $\mathrm{x}$ - and y-axis. Therefore, the k-point number along the z-axis is one half of the k-point number along $\mathrm{x}$ - and $\mathrm{y}$-axis to ensure approximately the same k-point density in each direction. A relatively high energy of $500 \mathrm{eV}$ was chosen as a plane wave cutoff to minimize the influence of Pulay stress during optimization. Geometry was optimized until the force on all individual atoms was less than $0.03 \mathrm{eV} / \AA$.

\subsection{Band structure and dielectric function calculations}

For ground state calculations a k-point grid of 40x40x40 k-points was used for 4-atom cells and 40x40x20 k-points for 8atom cells. Plane wave cutoff was set to $400 \mathrm{eV}$ and additional empty bands were used in the calculations. All parameters were tested for convergence. Based on these ground state calculations, band structure was calculated using the same number of bands as in ground state calculations for each system. The same ground state calculations were the starting point for dielectric function calculations, which were performed within the random phase approximation (RPA) framework and were based on the approach from [59]. Broadening parameter $\eta$ was set to $0.15 \mathrm{eV}$ and the rate parameter $\gamma$ was set to $0.01 \mathrm{eV}$ to all dielectric functions shown here in figures, while for electrodynamic simulations $\gamma$ was linearly interpolated between values from [45] for pure $\mathrm{Au}$ and $\mathrm{Ag}$ to avoid large discrepancies from data based on experiments in the IR.

\subsection{Electrodynamics simulations}

Experimentally determined optical constants used for comparision with DFT calculations and for electrodynamics simulations are taken from [10]. Electrodynamics calculations are performed using a variety of methods. Small particle polarizability, dispersion relations at glass/metal interface and reflectance of Kretschmann and air/metal configurations are analytically calculated with standard expressions that can be found elsewhere [60]. The near field properties of the dimer system are computed using an own implementation of the multiple-particle Mie theory [61]. The nanorod response is computed using the MNPBEM toolbox based on the boundary element method for the resolution of Maxwell equations in geometries that do not accept analytical solutions [62].

\section{Acknowledgments}

The authors thank the financial support of the Croatian Science Foundation through the grant number IP-2019-04-5424.

\section{Conflicts of Interest}

The authors declare that there is no conflict of interest regarding the publication of this article. 


\subsection{Author contributions}

M.B. performed the DFT calculations, while J.S.P. performed the electrodynamics calculations. Both authors performed data analysis and contributed to writing the manuscript, each focusing more on the parts associated with their respective calculations.

\section{Data Availability}

The data is available upon reasonable request.

\section{Supporting Information}

Supporting Information is available from the Wiley Online Library or from the author.

\section{References}

[1] S. Lal, S. Link, N. J. Halas, Nature Photonics 2007, 1 641-648.

[2] D. K. Gramotnev, S. I. Bozhevolny, Nature Photonics 2010, 483.

[3] S. Hayashi, T. Okamoto, Journal of Physics D: Applied Physics 2012, 45433001.

[4] H. Yu, Y. Peng, Y. Yang, L. Zhi-Yuan, npj Computational Materials 2019, 545.

[5] M. L. Brongersma, N. J. Halas, P. Nordlander, Nature Nanotechnology 2015, 1025.

[6] S. A. Maier, Plasmonics: Fundamentals and Applications, Springer, 2007.

[7] A. Moores., F. Goettmann, New Yournal of Chemistry 2006, 301121.

[8] S. Eustis, M. A. El-Sayed, Chemical Society Review 2005, 35209.

[9] B. Doiron, M. Mota, M. P. Wells, R. Bower, A. Mihai, Y. Li, L. F. Cohen, M. Alford, Neil, P. K. Petrov, R. F. Oulton, S. A. Maier, ACS Photonics 2019, 6240.

[10] O. Peña-Rodríguez, Journal of Alloys and Compounds 2017, 694857.

[11] D. Rioux, S. Vallières, S. Besner, P. Muñoz, E. Mazur, M. Meunier, Advanced Optical Materials 2013, 2176.

[12] M. R. S. Dias, M. S. Leite, Accounts of chemical research 2019, 522881.

[13] T. Gong, P. Lyu, K. J. Palm, S. Memarzadeh, J. N. M. Munday, M. S. Leite, Advanced Optical Materials 2020, 8 2001082 .

[14] A. M. Brown, R. Sundararaman, P. Narang, W. A. Goddard, H. A. Atwater, ACS Nano 2016, 10 957-966.

[15] T. Rangel, D. Kecik, P. E. Trevisanutto, G.-M. Rignanese, H. Van Swygenhoven, V. Olevano, Physical Review B 2012, 86125125 .

[16] R. Sundararaman, P. Narang, A. S. Jermyn, W. A. Goddard III, H. A. Atwater, Nature Communications 2014, 5 5788 .

[17] J. P. Perdew, K. Burke, M. Ernzerhof, Physical Review Letters 1996, 77 3865-3868.

[18] G. Prandini, G.-M. Rignanese, N. Marzari, npj Computational Materials 2019, 5129.

[19] L. J. Krayer, K. J. Palm, C. Gong, A. Torres, C. E. P. Villegas, A. R. Rocha, M. S. Leite, J. N. Munday, ACS Photonics 2020, 7 1689-1698.

[20] M. L. Brongersma, N. J. Halas, P. Nordlander, Chemical Society Review 2005, 35209.

[21] V. J. Keast, J. Ewald, M. B. De Silva, K. S. B.and Cortie, B. Monnier, D. Cuskelly, E. H. Kisi, Journal of Alloys and Compounds 2015, 647129.

[22] M. N. Gjerding, M. Pandey, K. S. Thygesen, Nature Communications 2017, 64715133.

[23] V. J. Keast, K. Birt, C. T. Koch, S. Supansomboon, C. M. B., Applied Physics Letters 2011, 99111908.

[24] V. J. Keast, R. L. Barnett, M. B. Cortie, Journal of Physics: Condensed Matter 2014, 26305501. 
[25] K. De Silva, A. Gentle, M. Arnold, V. Keast, M. Cortie, Journal of Physics D: Applied Physics 2015, 48215304.

[26] C. Gong, A. Kaplan, Z. A. Benson, D. R. Baker, J. P. McClure, A. R. Rocha, M. S. Leite, Advanced Optical Materials 2018, 61802185 .

[27] J. P. McClure, J. Boltersdorf, D. R. Baker, T. G. Farinha, N. Dzuricky, E. P. V. Cesar, A. R. Rocha, M. S. Leite, ACS Applied Materials \& Interfaces 2019, 11 24919-24932.

[28] K. Capelle, Brazilian Journal of Physics 2006, 36 1318-1343.

[29] E. I. Solomon, R. A. Scott, B. R. King, Computational Inorganic and Bioinorganic Chemistry, EIC Books. Wiley, 2009.

[30] P. Verma, D. G. Truhlar, Trends in Chemistry 2020, 2302.

[31] M. Swartf, M. F. Bickelhaupt, M. Duran, Dft2019 poll, URL https://www.marcelswart.eu/dft-poll/newsitem.pdf.

[32] N. Madriossian, M. Head-Gordon, Trends in Chemistry 2017, 1152315.

[33] I.-B. Lin, T. W.-H. Sheu, J.-H. Li, Optics Express 2014, 2230725.

[34] M. Kuisma, A. Sakko, T. P. Rossi, A. H. Larsen, J. Enkovaara, L. Lehtovaara, T. T. Rantala, Physical Review B 2015, 91115431.

[35] J. Yan, K. W. Jacobsen, K. S. Thygesen, Physical Review B 2011, 84235430.

[36] J. Yan, K. W. Jacobsen, K. S. Thygesen, Physical Review B 2012, 86241404.

[37] O. Gritsenko, R. van Leeuwen, E. van Lenthe, E. J. Baerends, Physical Review A 1995, 511944.

[38] M. Kuisma, J. Ojanen, J. Enkovaara, T. T. Rantala, Physical Review B 2010, 82115106.

[39] P. Haas, F. Tran, P. Blaha, Physical Review B 2009, 79085104.

[40] G. I. Csonka, J. P. Perdew, A. Ruzsinszky, P. H. T. Philipsen, S. Lebègue, J. Paier, O. A. Vydrov, J. G. Ángyán, Physical Review B 2009, 79155107.

[41] J. P. Perdew, Y. Wang, Physical Review B 1992, 4513244.

[42] J. P. Perdew, A. Ruzsinszky, G. I. Csonka, O. A. Vydrov, G. E. Scuseria, L. A. Constantin, X. Zhou, K. Burke, Physical Review Letters 1976, 100136406.

[43] B. Hammer, L. B. Hansen, J. K. Nørskov, Physical Review B 1999, 597413.

[44] M. Dion, H. Rydberg, D. C. Schröder, E.and Langreth, B. I. Lundqvist, Physical Review Letters 2004, 92246401.

[45] O. Peña-Rodríguez, M. Caro, A. Rivera, J. Olivares, J. M. Perlado, A. Caro, Optical Materials Express $2014,4403$.

[46] E. D. Palik, Handbook of optical constants of solids, Elsevier, 1998.

[47] P. B. Johnson, C. R. W., Physical Review B 1974, 95056.

[48] B. Himmetoglu, A. Floris, S. de Gironcoli, M. Cococcioni, International Journal of Quantum Chemistry 2013, 114 14.

[49] W. Köster, R. Stahl, Zeitschrift für Metallkunde 1967, 58 768-777.

[50] J. Rivory, , M. L. Theye, Journal de Physique Lettres 1975, 3629.

[51] H. P. Myers, L. Walldén, K. Å., The Philosophical Magazine: A Journal of Theoretical Experimental and Applied Physics 1968, 18725.

[52] L. K. Skriver, H. P. Lengkeek, Physical Review B 1979, 19900.

[53] J. Banhart, Physical Review Letters 1999, 82 2139.

[54] J. Rivory, Physical Review B 1977, 153119.

[55] J. J. Mortensen, L. B. Hansen, K. W. Jacobsen, Physical Review B 2005, 71035109. 
[56] J. Enkovaara, C. Rostgaard, J. J. Mortensen, J. Chen, M. Dułak, L. Ferrighi, J. Gavnholt, C. Glinsvad, V. Haikola, H. A. Hansen, H. H. Kristoffersen, M. Kuisma, A. H. Larsen, L. Lehtovaara, M. Ljungberg, O. Lopez-Acevedo, P. G. Moses, J. Ojanen, T. Olsen, V. Petzold, N. A. Romero, J. Stausholm-Møller, M. Strange, G. A. Tritsaris, M. Vanin, M. Walter, B. Hammer, H. Häkkinen, G. K. H. Madsen, R. M. Nieminen, J. K. Nørskov, M. Puska, T. T. Rantala, J. Schiøtz, K. S. Thygesen, K. W. Jacobsen, Journal of Physics: Condensed Matter 2010, 22, 25253202.

[57] A. H. Larsen, J. J. Mortensen, J. Blomqvist, I. E. Castelli, R. Christensen, M. Dułak, J. Friis, M. N. Groves, B. Hammer, C. Hargus, E. D. Hermes, P. C. Jennings, P. B. Jensen, J. Kermode, J. R. Kitchin, E. L. Kolsbjerg, J. Kubal, K. Kaasbjerg, S. Lysgaard, J. B. Maronsson, T. Maxson, T. Olsen, L. Pastewka, A. Peterson, C. Rostgaard, J. Schiøtz, O. Schütt, M. Strange, K. S. Thygesen, T. Vegge, L. Vilhelmsen, M. Walter, Z. Zeng, K. W. Jacobsen, Journal of Physics: Condensed Matter 2017, 29, 27273002.

[58] H. J. Monkhorst, J. D. Pack, Physical Review B 2008, 13 188-5192.

[59] J. Yan, J. J. Mortensen, K. W. Jacobsen, K. S. Thygesen, Physical Review B 2011, 83245122.

[60] L. Novotny, B. Hecht, Principles of nano-optics, Cambridge university press, 2012.

[61] J. Gérardy, M. Ausloos, Physical Review B 1982, 25, 64204.

[62] U. Hohenester, A. Trügler, Computer Physics Communications 2012, 183, 2370. 